\title{
Davydov Soliton Collisions
}

\author{
Benkui Tan \\ Department of Geophysics \\ Peking University \\ Beijing 100871 \\ People's Republic of China \\ Telephone: 86-10-62755041 \\ email:dqgchw@ibmstone.pku.edu.cn
}

\author{
John P. Boyd \\ Dept. Atmospheric, Oceanic and Space Science \\ University of Michigan \\ 2455 Hayward Avenue \\ Ann Arbor, MI 48109 \\ United States of America \\ email: jpboyd@engin.umich.edu
}

January 6, 1998

\begin{abstract}
In this paper, we study the collisions of Davydov solitons. The collision behavior is diverse and complicated, very sensitive to both the initial phases and velocities of the solitons. For some parameter ranges, Davydov solitons are stable to collisions in the sense that the solitons retain their structures, though for some cases, the propagation direction may be altered. For other parameter ranges, significant structural changes may occur: An exchange of energy between solitons, or the merger of two solitons to form a new bound state.

Keywords: Molecular soliton, Davydov soliton, soliton interactions
\end{abstract}


The propagation of solitons in certain types of molecular chains, e. g., the so-called $\alpha$-helix, has been modeled by the so-called Davydov chain equations [2]:

$$
\begin{aligned}
i \frac{\partial A}{\partial t}+\frac{\partial^{2} A}{\partial x^{2}} & =A B \\
\frac{\partial^{2} B}{\partial t^{2}}-\frac{\partial^{2} B}{\partial x^{2}} & =\frac{\partial^{2}}{\partial x^{2}}\left(|A|^{2}\right)
\end{aligned}
$$

where $A$ is the wave probability amplitude and $B$ is the longitudinal displacement. There is controversy, as with most simple models, as to the accuracy of this model for molecular chains. However, the same model is the onedimensional Zakharov equations for Langmuir solitons in plasmas. The model is also interesting because it reduces to both the Nonlinear Schrödiner equation and to the Long Wave/Short Wave Resonance equations in the appropriate limit.

Although the Davydov chain equations are not integrable by the inverse scattering method, they nevertheless have exact analytical solutions of the form

$$
\begin{aligned}
& A=\sqrt{2\left(1-4 \xi^{2}\right)} \eta \operatorname{sech}\{\eta(x-2 \xi t)\} \exp \left\{i \xi x-i\left(\xi^{2}-\eta^{2}\right) t+i \phi\right\}, \\
& B=-2 \eta^{2} \operatorname{sech}^{2}\{\eta(x-2 \xi t)\} .
\end{aligned}
$$

This is the "Davydov soliton". It consists of two parts: the wave probability amplitude $A$ is an envelope solitary wave and the longitudinal displacement $B$ is a "bell" soliton. The Davydov soliton depends on three parameters: $\eta, \xi$, and $\phi . \eta$ is related to the amplitude and width of the soliton, $\xi$ is half the velocity of the solitary wave, and $\phi$ is the phase of the sinusoidal factor of $A$ at $t=0$.

The Davydov soliton has been intensively studied, such as Davydov and Kislukh[2], who show that such solitary waves are stable to small perturbations. However, no previous work on soliton-soliton collisions for this system is known to us. The goal of the present study is investigate such collisions.

We solve Eqs.(1) by the Fourier pseudospectral method[1, 3] using 256 basis functions for the spatial discretization combined with fourth order Runge-Kutta for time-marching. The Davydov system has two exact time-independent energy invariants:

$$
E_{1} \equiv \int_{-\infty}^{\infty}|A|^{2} d x, \quad E_{2} \equiv \int_{-\infty}^{\infty} B d x
$$

These are helpful to check the accuracy of the numerical calculation. (When the solitons are well-separated, we can evaluate the contributions of each soliton to these invariants with an error which is exponentially small in the separation between the two solitons; numerical values are given in the figure captions.) For the collision experiments, the initial condition is the sum of two solitary waves separated by a distance $x_{0}$ :

$$
A=\sqrt{2\left(1-4 \xi_{1}^{2}\right)} \eta_{1} \operatorname{sech}\left(\eta_{1} x\right) \exp \left\{i \xi_{1} x+i \phi_{1}\right\}
$$




$$
\begin{aligned}
& +\sqrt{2\left(1-4 \xi_{2}^{2}\right)} \eta_{2} \operatorname{sech}\left\{\eta_{2}\left(x+x_{0}\right)\right\} \exp \left\{i \xi_{2}\left(x+x_{0}\right)+i \phi_{2}\right\}, \\
B= & -2 \eta_{1}^{2} \operatorname{sech}^{2}\left\{\eta_{1} x\right\}-2 \eta_{2}^{2} \operatorname{sech}^{2}\left\{\eta_{2}\left(x+x_{0}\right)\right\} .
\end{aligned}
$$

where the first terms in each equation are soliton 1 while the rest are soliton 2. The graphs show that the post-collision fate of the solitary waves is strongly dependent on both the initial phases and the initial velocities of the solitons. Since $A$ can be multiplied by $\exp (i \phi)$ where $\phi$ is an arbitrary constant and still remain a solution (with $B$ unchanged), one of the phases is arbitary: only the relative difference in the two initial phases is significant. So, without loss of generality, we set $\phi_{1}=0$ in all cases.

Figs. 1 shows the fast collisions in which the initial relative velocity between the two solitons is fixed to be 1.8. The relative phases increase from 0 (top) to $\pi / 2$ (middle) to $\pi$ (bottom).

When the relative phase is zero, (top pair of plots), the solitons freely penetrate each other but emerge with their shapes and velocities unchanged. In contrast, when $\phi_{2}=\pi / 2$ (middle graphs), $A$ emerges from the collision very asymmetrically - a large solitary wave moving to the left (at the same velocity as the initial speed of soliton 2) plus a small pulse travelling to the right at the speed of the original soliton 1 . The corresponding $B$ components differ much less. The bottom pair of contour plots show what happens when $\phi_{2}=\pi$. The $B$-components freely penetrate, but the $A$-components bounce off each other with a change of direction but no merger or interpenetration.

The fourth case, $\phi_{2}=3 \pi / 2$, is not illustrated because it is just the mirror image of the middle figure. That is,

$A\left(x, t ; \phi_{2}=3 \pi / 2\right)=A\left(-x, t ; \phi_{2}=\pi / 2\right), \quad B\left(x, t ; \phi_{2}=3 \pi / 2\right)=B\left(-x, t ; \phi_{2}=\pi / 2\right)$

The same is true for intermediate and slow collisions also. The reflection principle cannot be generalized to all solutions differing in initial phase by $\pi$ because the cases $\phi_{2}=0$ and $\phi_{2}=\pi$ are in fact quite different.

The next four cases are identical to the first four except that initial collision velocity is 1 , which is intermediate. When $\phi_{2}=0$, the solitons reform after a fast collision, but at a medium velocity, the solitons merge to form a permanent, stationary bound state (top pair in Fig. 2). Small amplitude remnants propagate away from the bound state both to the left and the right, but the bound state absorbs most of the energy of the two initial solitary waves. When the relative phase is increased to $\pi / 2$, the collision is highly asymmetrical, just as at higher collision velocity. However, the bound state propagates leftward more slowly than soliton 2 whereas it matched the speed of soliton 2 at higher collision velocity. When the relative phase of the colliding solitary waves is increased to $\phi_{2}=\pi$, the solitons reflect each other, closely approaching but never interpenetrating, just as happened at this phase for fast collisions. As before, a relative phase of $3 \pi / 2$ is not illustrated because this is identical with the middle plots for $\phi_{2}=\pi / 2$ except for the interchange $x \rightarrow-x$. 
Fig. 3 illustrates slow collisions where the initial collision velocity was only 0.2. When the relative phase is either 0 or $\pi$ [top and bottom graphs], the qualitative behavior is very similar to the intermediate collisions for these same phases: formation of a stationary bound state at $\phi_{2}=0$, repulsion without merging at $\phi_{2}=\pi$. However, at a relative phase of $\pi / 2$ (and its mirror image at $3 \pi / 2$, not illustrated), all is changed. Soliton 1 is completely destroyed: Part of its energy is reflected but part is captured by soliton 2. After gaining the energy, soliton 2 becomes stationary and much stronger. Some small remnants are also radiated away from the collision zone.

In conclusion, the collisions of Davydov solitons exhibit reflection, mergerand-reforming, merger-to-a-bound state, and partial destruction, all depending on the initial relative phase and velocity.

This work was supported by NSF grant OCE9521133. We thank the reviewers for useful comments.

\section{References}

[1] J. P. Boyd, Chebyshev and Fourier Spectral Methods, Springer-Verlag, New York, 1989. $792 \mathrm{pp}$.

[2] A. S. Davydov and N. I. Kislukha, Soviet Physics JETP, 44 (1976), p. 571.

[3] B. F. Sanders, N. Katopodes, and J. P. Boyd, Unified pseudo-spectral solution to water wave equations, J. Engin. Hydraulics of. ASCE, (1998). In press;. 

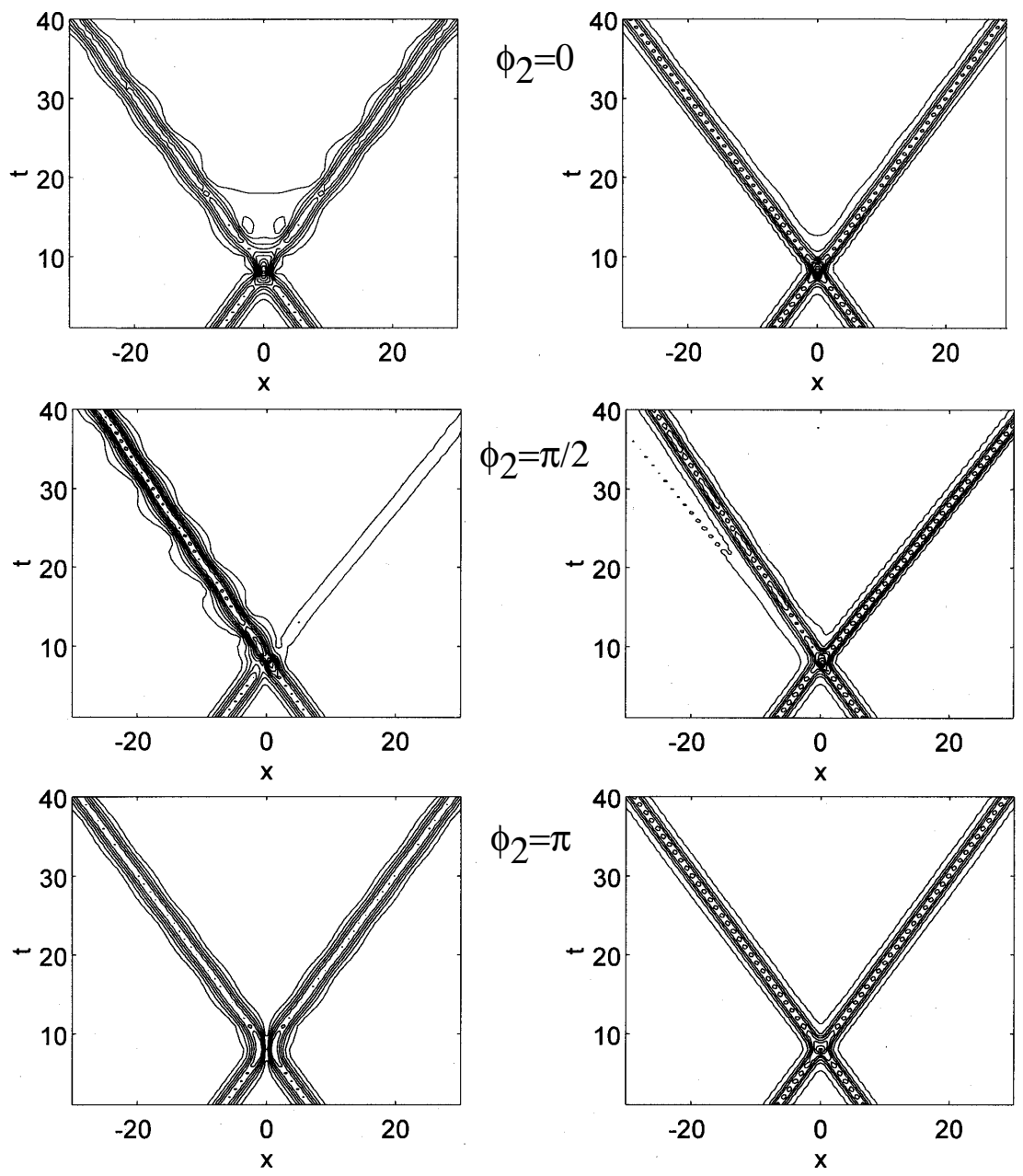

Figure 1: FAST COLLISIONS, relative velocity of 1.8. All cases are identical except that $\phi_{2}$ is increased in increments of $\pi / 2$ beginning with $\phi_{2}=0$ at the top. Contours of the absolute value of the wave probability amplitude $A$ is on the left in each pair of plots with $x$ as the horizontal coordinate and $t$ increasing upward; isolines of absolute value of $B$ are illustrated on the right of each pair of graphs. The fourth case, $\phi_{2}=3 \pi / 2$ is not shown because it is just the mirror image of the middle pair of plots. Before the collision, each of the initial solitons contributes 0.7600 to $E_{1}$ [for all three cases: top, middle, and bottom]. In the middle left plot, after the collision, the larger contributes 1.4272 while the smaller gives 0.0928. (The post-collision energies are the same as the precollision energies for $A$ when $\phi_{2}=0$ and $\phi_{2}=\pi$.) For all values of $\phi_{2}$, there is little change in the contributions of the solitons in their $B$-field to the energy $E_{2}$, so we do not list numerical values for these contributions. 

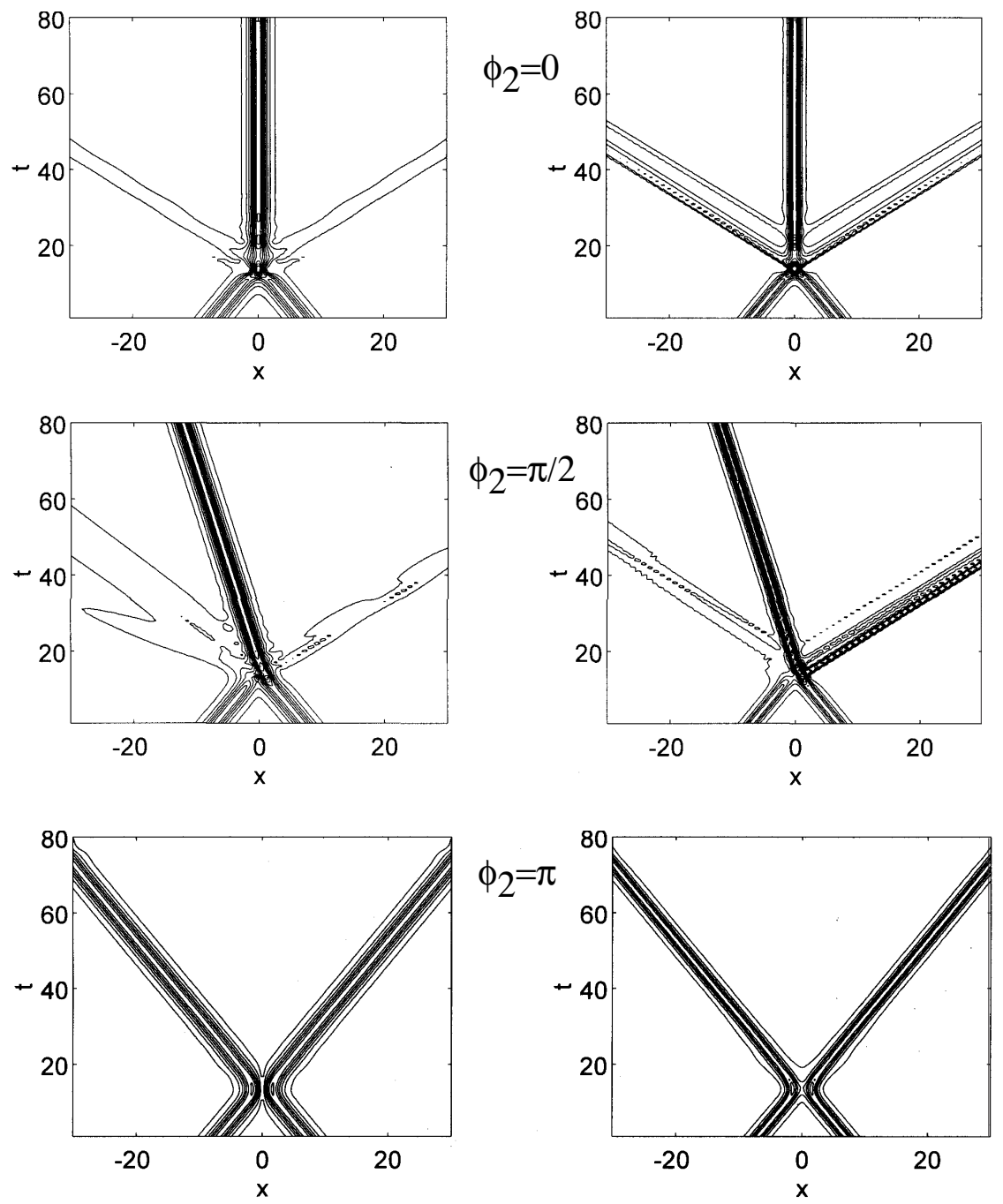

Figure 2: Contours of absolute value of $A$ (left plot in each pair) and $B$ (right), same as Fig. 1 except for INTERMEDIATE COLLISIONS, relative velocity of 1 . For all three cases, the initial energy of each $A$ soliton is 3.0000 while each $B$ soliton has $E_{2}=-4.0000$. In the top right figure, the energies of the three emerging solitary waves are, from left to right, $0.2261,5.5612$, and 0.2130 ; the post-collision $E_{2}$ of the $B$ solitons are $-1.2296,-5.5815$, and -1.1922 . For the middle pair of graphs, the after-collision energy of the $A$ soliton is 5.1547 while the two patches of dispersing transients collectively contribute 0.8453 ; the $B$ soliton has $E_{2}=-5.3566$ while the left and right patches of remnants contribute -0.9522 and -1.6919 , respectively. In the bottom case, $\phi_{2}=\pi$, the final energies of the solitons are the same as the initial energies. $\left(\phi_{2}=3 \pi / 2\right.$ is not shown because it is just the mirror image of $\phi_{2}=\pi / 2$.) 

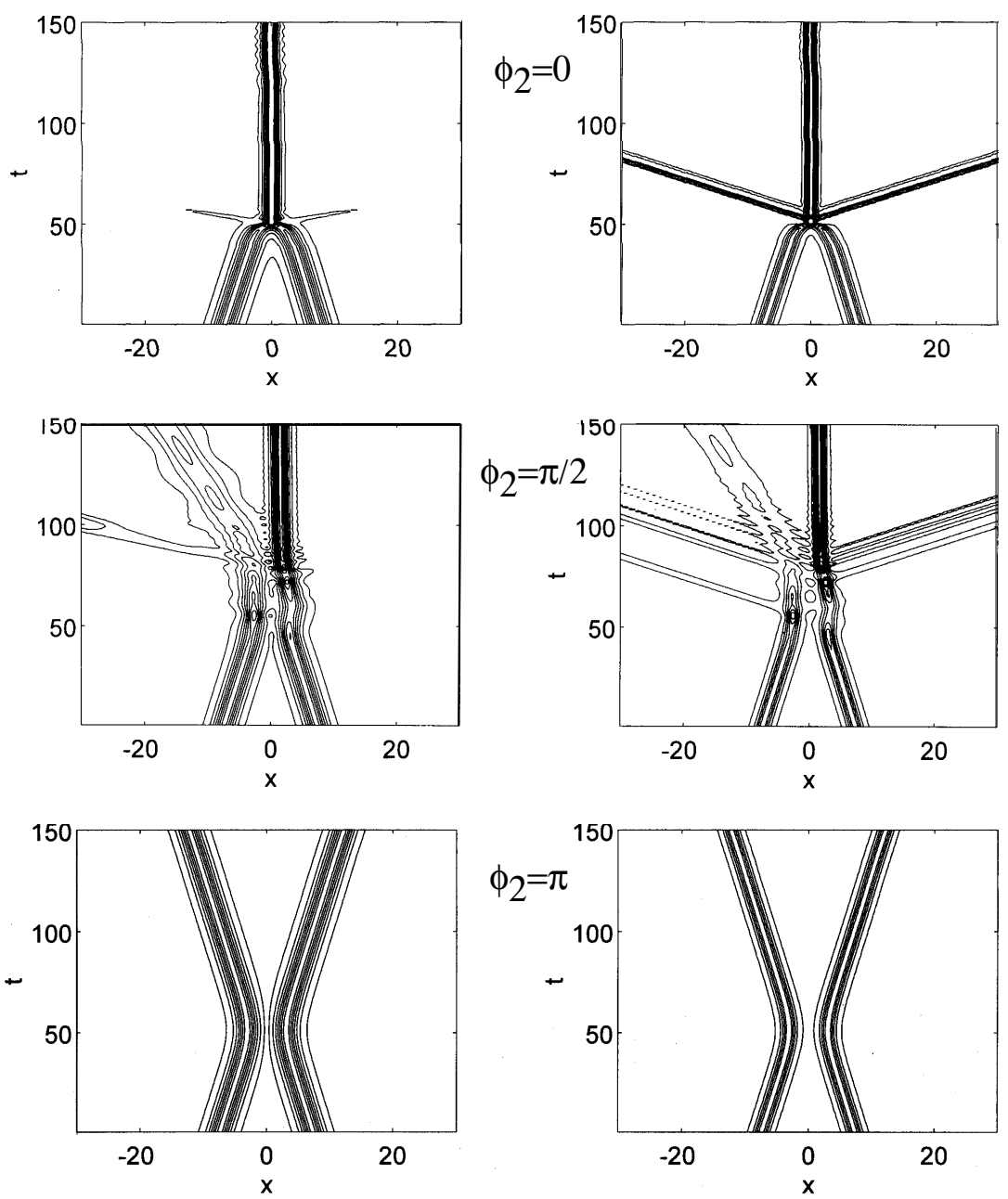

Figure 3: Same as Fig. 1 except for SLOW COLLISIONS, relative velocity of 0.2 . The initial $E_{1}$ of each $A$ soliton is 3.9600 while each $B$ solitary waves contributes -4.0000 to $E_{2}$. After the collision in the top left figure, the single large $A$ solitary wave has $E_{2}=7.8404$ while the two offshoots contribute only 0.0796 together; the emergent $B$ solitary wave has $E_{2}=-7.8355$ to $E_{2}$ while the left and right smaller pulses contribute -0.0204 and -0.1498 , respectively. For the middle graphs $\left(\phi_{2}=\pi / 2\right), E_{1}$ of the two post-collision $A$ solitary waves are 5.7195 and 2.0756 while the two $B$ solitons contribute -5.6789 and -2.0167 ; the non-solitonic remnants contribute 0.1249 to $E_{1}$ and -0.6047 to $B$. For the bottom case, the invariants of the initial and final solitary waves are the same for both $A$ and $B$. $\left(\phi_{2}=3 \pi / 2\right.$ is not shown because it is identical with $\phi_{2}=\pi / 2$ with the substitution $x \rightarrow-x$ (mirror image).) 